high-frequency medical apparatus. Amongst small commercial apparatus are refrigerators, coffee grinders, dental drills, hair driers and clippers. Amongst large apparatus are generators, motors, flashing signs, traffic signals and overhead transmission lines. The interference due to traction plant for trains, trolley buses and electric trams is often serious. The petrol engines of automobiles and aircraft with coil or magneto ignition sometimes cause trouble. Experience shows that the amount of radio interference is becoming greater and its distribution more widespread. The remedy can be applied by the radio engineer in many cases at the receiving set, but the general level of the disturbance can be controlled only by suitable devices at the source. The presentday trouble is not primarily an electrical one, but is to find out a method of reconciling the economic aspects of the various interests concerned.

\section{Physical Investigations of Psychical Phenomena}

Ir has been suggested on several occasions that the time has come for the critical and objective study of certain psychical ('para-normal') phenomena by the accredited experimental methods of physics, physiology and psychology. How these methods may be applied to such a problem as, for example, telekinesis (the movements of objects without physical contact but in presence of an entranced 'medium') has been investigated in Paris by Dr. Eugène Osty, working with infra-red rays (see NATURE, November 25, p. 801). These curious effects have also been studied by $\mathrm{Mr}$. Harry Price at his National Laboratory of Psychical Research in London. Fortunately, research into such para-normal happenings requires no belief in the truth or falsity of spiritualism; it is the outcome of the unprejudiced study of very special phenomena by the methods of the modern laboratory. Prof. Fraser-Harris, writing from The Athenæum, Pall Mall, S.W.1, informs us that an effort is to be made to endow and equip an institute for the critical study of psychical phenomena by the objective methods of registration. The promoters of the scheme realise that such things as the nature of the trance-state of a teledynamist, the 'direct voice', and materialisations ('ectoplasm') are now amenable to be investigated by delicate instruments and by exquisite methods which were non-existent a generation ago. Photography by ultraviolet light and by infra-red rays, the reception and transmission of sounds and voices by the microphone and gramophone, are sufficient to indicate that science is equipped as never before to attack problems apparently the most mysterious. It is hoped that funds may be forthcoming to make it possible to endow and equip an institute of psychical research worthy of the importance of the subjects to be investigated. Our own view, however, is that such an institute should be attached to the psychological department of a university or college, or to a responsible scientific society, and not be under the control of a private governing body. If established under such auspices it might maintain the reputation of Great Britain as the traditional home of genuine, unfettered and fearless research.

\section{Pictorial Representation of Data}

ONE of the characteristics of scientific management in modern industry is the use which is made of graphical methods. The importance of the pictorial representation of facts and data has also been widely realised by the various movemonts aiming at the prevention of accidents whether in industry or in the streets. It is, however, only within the last ten years that pictorial representations have been fashioned on definite scientific principles, and the value of the pioneer work of the Mundaneum Institute, Vienna, is now becoming widely recognised. During the last decade, under the leadership of Dr. Otto Neurath, basic principles for visual presentation have been developed. Charts or illustrations constructed on these lines reveal what is most essential at a first glance; the important details stand out on a second glance and more exact details are evident to a third glance. The method has been applied with conspicuous success to technical and to social facts and data, and the work of the Mundaneum has become known through a series of publications such as Gesellschaft und Wirtschaft, Technik und Menschhiet, Die Bunte Welt and Bildstatistik. Branches have now been established in Amsterdam and London (c/o World Association for Adult Education, 16, Russell Square, W.C.1) through which the services offered, including the preparation of charts, the loan of exhibits, issue of publications and provision of material, and advice on principles of visual presentation may be more accessible. The new technique provides an international cultural factor of high importance, but if its full advantages are to be reaped, its introduction into different countries should proceed on uniform lines under the guidance of the Mundaneum itself.

\section{Unemployment and Training Schemes}

AN article on "Training and Unemployment" by Mr. Morris S. Viteles appears in the Human Factor, 7, No. 9. Mr. Viteles points out that the feeling of economic insecurity consequent upon unemployment and fear of unemployment is responsible for a great deal of the individual maladjustment in industry at the present time. Training schemes applied to entrants have tended to increase their efficiency and stability. At the same time, injustice has been done to workers of many years' standing, who are not given the benefit of this aid to efficiency, on the assumption that their experience makes it unnecessary, and that they would resent it. The writer advocates the training of older workers as well as new, and also the incorporation of instruction in allied tasks and processes into every training scheme-so that the mobility and adaptability of the workers within any given organisation unit may be at a maximum. Considerable increase in the worker's sense of security would follow, since he is good for more than one job. The chief psychological problems involved are those concerning the nature of motor skills and the probability of a general underlying factor; the transference of skill; and the possibility of accurately 